\title{
Morphological Alterations of CAD Cells Overexpressing AKT1
}

James Wachira

Morgan State University, Baltimore, Maryland, United States

\section{Abstract}

CAD cells are neuronal cells used in studies of cell differentiation and in cellular models of neuropathology. When cultured in differentiation medium, CAD cells exhibit characteristics of mature neurons including the generation of action potential. In addition to being a central signaling kinase in cell survival, AKT1 plays important roles in the nervous system including neuroplasticity and this study examined the localization of exogenous AKT1 in CAD cells. Neuropeptides modulate many signal transduction pathways and melacortins are implicated in regulating growth factor signal transduction pathways, including the PI3K/AKT pathway. AKT1-DsReD was transfected into CAD cells that were stably expressing melanocortin 3-receptor-GFP (MC3R-GFP), a G-protein coupled receptor. The cells were imaged with confocal microscopy to determine the fluorescent protein localization patterns. AKT1DsRed was predominantly localized in the cytoplasm and the nucleus. Further, expression of exogenous AKT1 in these cell lines led to morphological changes reminiscent of apoptosis. As expected, MC3R-GFP localized to the plasma membrane but it internalized upon cell stimulation with the cognate ligand. In limited areas of the plasma membrane, AKT1-DsRed and MC3R-GFP were colocalized. In conclusion, quantitative studies to understand the role of relative levels of AKT1 in determining cell survival are needed.

\section{Introduction}

CAD cells undergo differentiation into a neuronal-phenotype with a large number of processes upon culture in serum free medium supplemented with insulin-transferrin-selenium (ITS)(Li et al., 2005). However, the differentiated cells exhibit heterogeneous morphologies with some extending long processes while others exhibit less extensive, thick processes while others are polynucleated(Li et al., 2005) . Whereas the molecular basis for the morphological heterogeneity remains uncharacterized, the cells appear to be more homogenous molecularly as they express many neuronal and glial markers including GAP43, a marker of neuronal growth cones, and glial fibrillary acidic protein (GFAP)(Li et al., 2005). CAD cells exhibit characteristics of neural progenitors that allow for the modeling of lineage specification of neuronal precursors( $\mathrm{Li}$ et al., 2005). They are very susceptible to infection by prion proteins and the differentiation of CAD 5 clone is associated with differential processing of PrPC, suggesting they could serve as a model for delineating molecular mechanisms underlying the generation and propagation of pathological prion proteins(Fremuntova et al., 2020).

The PI3K/AKT pathway regulate cell growth and proliferation downstream of growth factor receptors(Hers et al., 2011). In the P19 cell line model of neuronal differentiation, PI3K/AKT/GSK3 $\beta$ signaling pathway is implicated the expression of makers of neuronal phenotype(Fu et al., 2020). It is therefore of great interest to understand how the PI3K/AKT signal transduction pathway affects the development of neuronal functions. 
Materials and Methods

\section{Plasmid construction}

Human AKT1 gene (GenBank gene ID: 11651) was cloned in-frame with DsRed fluorescent protein gene in pDsRed2-N1 monomer vector (BD Biosciences). The MC3R-GFP construct has been published(Nyan et al., 2008).

\section{Cell culture}

CAD cells were cultured following standard mammalian cell culture techniques in DMEM/F12 medium supplemented with $8 \%$ heat inactivated fetal bovine serum (FBS). Differentiation was induced by culturing the cells in serum-free medium supplemented with insulin, transferrin and selenium. They were transfected at 40-60\% confluency with plasmid DNA prepared in fugene reagent following the manufacturer supplied protocol (Roche Applied Science).

\section{Confocal Microscopy}

The cells were cultured on cover-glass and then imaged. The laser power was adjusted as per recommendations to reduce bleed-through between different channels. The cells were imaged with a Nikon TE2000-E laser scanning confocal microscope (Nikon USA).

Results and Discussion

Whereas melanocortin receptors are coupled to the cAMP/PKA pathway, many laboratories have reported that they also signal through growth factor signaling pathways, including the Ras/MAPK and PI3K/AKT pathways(Yang \& Tao, 2017). Activation of secondary pathways by G-protein-coupled receptors (GPCRs) is attributed to recruitment of arrestins, which also play a role in receptor desensitization(JeanCharles et al., 2017). The regulation of melanocortin receptors by arrestins has also been reported(Nyan et al., 2008). Expression of AKT1-DsRed in MC3R-GFP expressing cells showed limited colocalization of these proteins (Figure 1). Further, whereas stimulation of M3R-GFP with $\gamma$-MSH, the endogenous agonist, led to its internalization, there were no changes in the localization of AKT1-DsRed. AKT1-DsRed was mostly localized in the intracellular compartment. Also observed were morphological changes in AKT-DsRed expressing cells that are consistent cell death, including shrinkage (Figure 2). These studies suggest that high levels of AKT1 may have adverse effects on cell survival that merit further characterization.

"Research reported in this publication was supported by the National Institute of General Medical Sciences of the National Institutes of Health under Award Number UL1GM118973. The content is solely the responsibility of the authors and does not necessarily represent the official views of the National Institutes of Health."

"We thank the National Institutes of Health for financial support through the cooperative agreement NIMHD 1U54MD013376-01A1." 


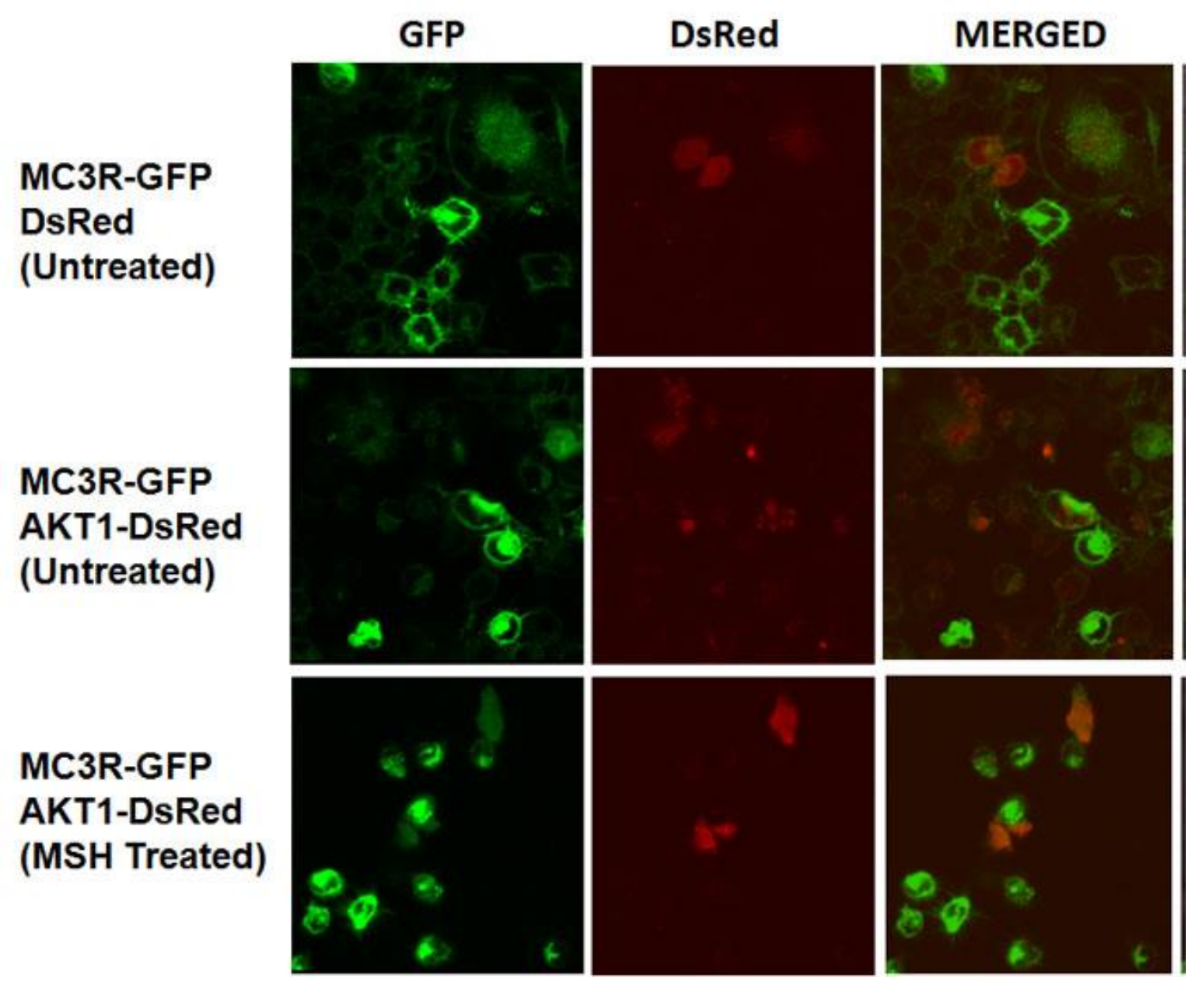

Figure 1. AKT1-DsRed appears as punctate dots in the cytoplasm and nucleus. The cells were transfected as indicated and either treated with $\gamma$-MSH or left untreated. 

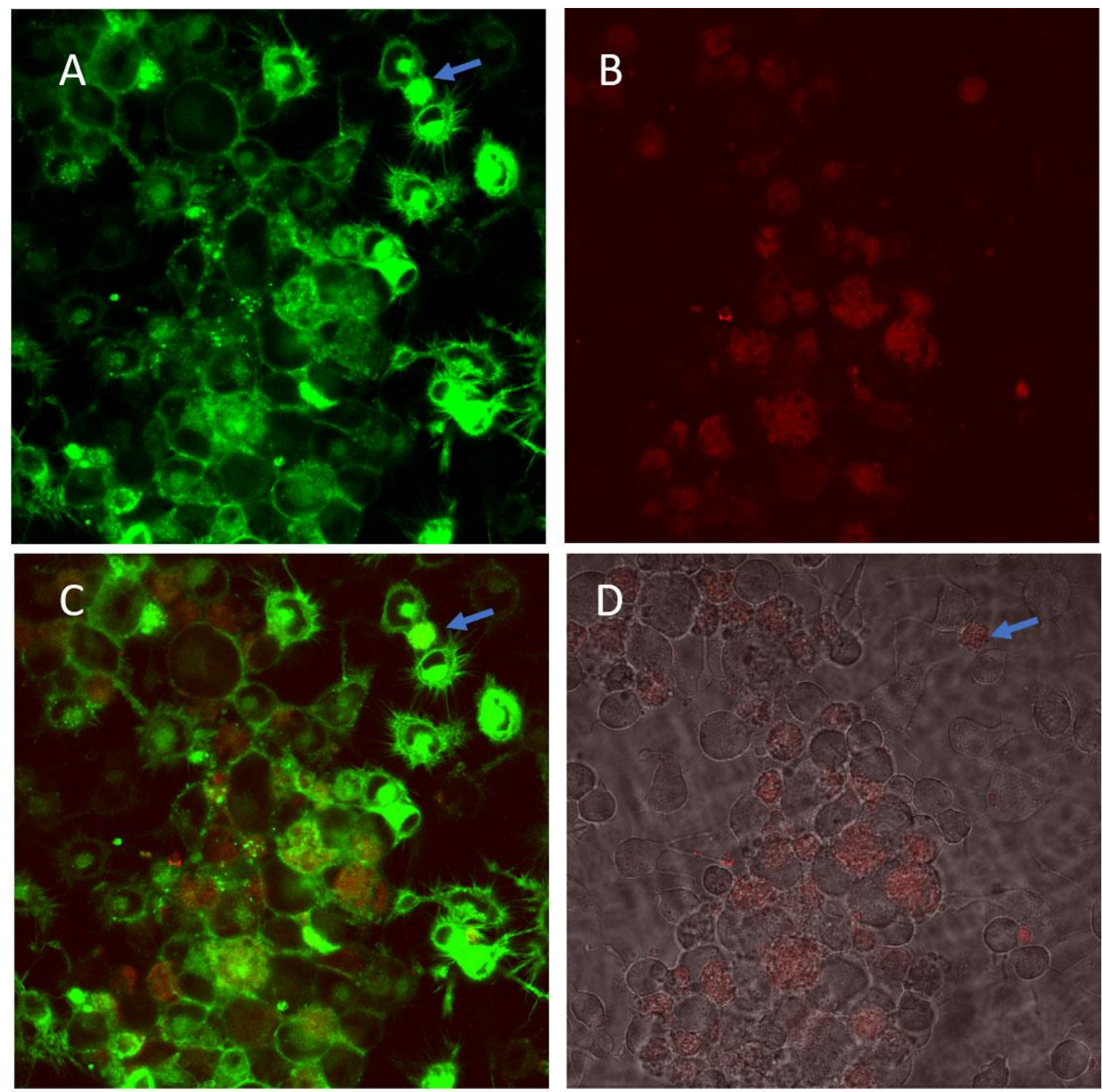

Figure 2. Morphological changes associated with AKT1-DsRed transfection. MC3R-GFP expressing cells were transfected with AKT1-DsRed, differentiated and imaged. A) MC3R-GFP is predominantly localized on the plasma membrane; B) and D) AKT1-DsRed is localized. The cells expressing AKT1DsRed appear smaller than neighboring cells (blue arrows). D) In the overlay image, there is limited overlap of the green and red signals indicating lack of colocalization.

\section{References}

Breit, A., Wolff, K., Kalwa, H., Jarry, H., Büch, T. \& Gudermann, T. (2006). The natural inverse agonist agouti-related protein induces arrestin-mediated endocytosis of melanocortin-3 and -4 receptors. The Journal of Biological Chemistry 281, 37447-37456.

Fremuntova, Z., Mosko, T., Soukup, J., Kucerova, J., Kostelanska, M., Hanusova, Z. B., Filipova, M., Cervenakova, L. \& Holada, K. (2020). Changes in cellular prion protein expression, processing and localisation during differentiation of the neuronal cell line CAD 5. Biology of the Cell 112, 1-21. 
Fu, F., Li, L.-S., Li, R., Deng, Q., Yu, Q.-X., Yang, X., Pan, M., Han, J., Zhen, L., Zhang, L.-N., Lei, T.Y., Li, D.-Z. \& Liao, C. (2020). All-trans-retinoid acid induces the differentiation of P19 cells into neurons involved in the PI3K/Akt/GSK3 $\beta$ signaling pathway. Journal of Cellular Biochemistry.

Hers, I., Vincent, E. E. \& Tavaré, J. M. (2011). Akt signalling in health and disease. Cellular Signalling 23, 1515-1527.

Jean-Charles, P.-Y., Kaur, S. \& Shenoy, S. K. (2017). GPCR signaling via $\beta$-arrestin-dependent mechanisms. Journal of cardiovascular pharmacology 70, 142-158.

Li, Y., Hou, L. X.-E., Aktiv, A. \& Dahlström, A. (2005). Immunohistochemical characterisation of differentiated CAD cells: expression of peptides and chromogranins. Histochemistry and Cell Biology 124, 25-33.

Nyan, D. C., Anbazhagan, R., Hughes-Darden, C. A. \& Wachira, S. J. M. (2008). Endosomal colocalization of melanocortin-3 receptor and beta-arrestins in CAD cells with altered modification of AKT/PKB. Neuropeptides 42, 355-366.

Yang, L.-K. \& Tao, Y.-X. (2017). Biased Signaling at Neural Melanocortin Receptors in Regulation of Energy Homeostasis. Biochimica et biophysica acta 1863, 2486-2495. 